\title{
Quantitative Analytical Model of the Formation Damage by Gel Particles
}

\author{
Mahmoud Elsharafi ${ }^{1, *}$, Laila Saleh $^{2}$ and Baojun Bai ${ }^{3}$ \\ ${ }^{1}$ McCoy School of Engineering, Midwestern State University, Wichita Falls, Texas, USA \\ ${ }^{2}$ Department of Petroleum Engineering, University of Tripoli, Tripoli, Libya \\ ${ }^{3}$ Department of Geosciences and Geological and Petroleum Engineering, Missouri University of Science and \\ Technology, Rolla, Missouri, USA
}

\begin{abstract}
Formation damage by gel particles has become one of the most important problems in mature reservoirs. The objective of the quantitative analytical model is to identify an analytical model to the best fit of the preformed particle gels (PPGs) filtration test results. This work will analyze the experiments results of low permeability core samples to evaluate the effect of various brine concentrations and particle sizes. This study used a linear analytical model relationship between cumulative volumes versus filtration time with a good fits result. Linear curve equations for the best fitting equation was obtained. According to quantitative analytical model for all of our filtration tests, the cumulative filtration test volume (Vcf) was explained in this paper. Quantitative analytical model results showed the value of the slop $\mathrm{m}$ increases as the injection pressure increases. Compared with the experiments, the results show that, if the value of the intercept $b$ $>2$ the damage occurred because the gel particles invasion started into the core surface. Results from the quantitative analytical model were indicated to have a good fitting with almost all of the experimental results. It is the first time to use quantitative analytical model to analysis the formation damage by the PPGs. The results can be used to select the best gel treatment design.
\end{abstract}

Keywords: Quantitative Analytical Model, Formation Damage, Conformance Control treatments Design, Preformed Particle Gels, Mature Reservoirs.

\section{INTRODUCTION}

A filtration test is a simple means of evaluating formation damage [1, 2]. The oil industry currently uses two standard filtration tests both static and dynamic, to assess damage to core samples. The former is suitable when testing for injection into the matrix rock; the latter assesses injection into a fracture [3]. Filtration test experiments have been used in the past to study the damage of cores fully saturated with brine, oil, or residual oil while injecting suspended particles, oily water, or a combination of both in these cores [4-7]. Elsharafi and Bai 2012 2013, 2015, and 2016 [8-11] studied the effect of deformable swollen gel particles on low-permeability zones. This research used static filtration tests experiments results to determine whether or not swollen PPGs affected unswept oil zones/areas. In addition, a filtration test was used to find methods for minimizing PPG damage. This research determined the better fits to the previous lab results [8-11]. The work has been conducted to determine the effect of preformed particle gels on unwept low permeable zones/areas. These results were obtained from an experimental work. filtration test model was used to find out the effect of gel particles on the formation damage.

*Address correspondence to this author at the McCoy School of Engineering, Midwestern State University, Wichita Falls, Texas, USA; Tel: 940-397-4551; Fax: 940-397-4536; E-mail: mahmoud.elsharafi@mwsu.edu
The effect of brine concentrations, particle sizes, gel types, and the rock permeability on the core damage were considered.

The primary objective of the quantitative analytical model is to identify an analytical model to the best fit of the PPG filtration test results obtained and analyze when the PPGs damage the cores. The volume versus square-root-of-time data for filtration tests is effectively described by Equation (1), first developed by Outmans (1963) [12] for drilling muds'.

$V f I=V s p+m \sqrt{ }$

Where, $\mathrm{Vfl}$ is the cumulative fluid-loss volume, Vsp is the spurt volume, and $\mathrm{m}$ is the slope of the linear part of the curve.

Barkman and Davidson (1972) [13] included the effect of solid particle invasion for static filtration tests. They noted that, if $b<0$, no damage has occurred. If $b>0$, the cores have been damaged. Variable $b$ is the intercept of the straight line which was used to determine whether or not damage has occurred.

Barkman and Davidson (1972) [13] suggested that the invasion of the solid particles takes place during the early part of the filtration test. They derived a simple equation during a linear filtration test. Their study included the cumulative volume (VB) at the bridging 
time $\left(\sqrt{ }\left(t_{-} B\right)\right)$. They also indicated that a plot of cumulative volume versus square root of time should produce a straight line when $\mathrm{t}>\mathrm{tB}$.

$V B=b+m \sqrt{ }\left(t \_B\right)$

Where, $b$ is the intercept of the straight line and $m$ is the slop of the straight line.

Gulbis (1983) [14] proposed using time rather than the square root of time. He displayed his result in Equation 3. His model provides a good fit with dynamic data taken from dynamic fluid-loss tests. Gulbis (1983) [14] used a hollow-core device.

$\mathrm{Vf} I=V s p+m t$

Roodhart (1985) [15] proposed the use of both time and square-root-of-time for dynamic fluid loss. Roodhart (1985) [15] used poloymetric Equation 4. This Equation includes both kick-building phase with a short time and an equilibrium flow region with a longer time.

$\mathrm{Vfl}=\mathrm{Vsp}+\mathrm{m} \sqrt{ }(\mathrm{t})+\mathrm{Bt}$

The constant (B) is, essentially, a fitting parameter that relates to the equilibrium flow region.

Penny et al. (1985) [16] introduced the power law model. They added the exponential tn rather than $t$. Thus, equation (5) fits well with curves that have a longer time.

$\mathrm{Vfl}=\mathrm{Vsp}+\mathrm{m}$ tn

Bourgoyne et al. (1986) [17] indicated that the preferred filtration test plot of a cumulative filtration loss versus the square root of time should be a straight line passing through the origin point when no spurt loss occurred. Some spurt loss, however, will always occur. This occurrence shifts the curve vertically, indicating that the intercept is not equal to zero.

Chin (1995) [18] determined that, for small wellbores diameter the square-root-of-time relationship cannot be used because of the effect of radial flow. The linear flow theory essential in the conventional analysis cannot be used.

Logeron et al. (1995) [19] used both the relationship between the cumulative filtration volume versus time and the cumulative filtration volume versus square root of time to determine particle invasion. Logeron et al. (1995) [19] used long cores for static filtration test. The relationship between the cumulative filtration volume versus the square root of time for static filtration tests indicates that, after a few minutes, the filtration tests curves almost a liner. Equation 6 describes the filtrate volume.

$V f=b+m \sqrt{t}$

Where, both $\mathrm{b}$ and $\mathrm{m}$ are constants which are affected by mud, core properties, and filtration test parameters.

Many researchers have used the square root of time, typically with dynamic filtration tests, and long cylindrical core samples. Long cores have been used to have a sufficient time to flow the mud filtrate invasion before filtrate break through.

This study could not obtain a linear function for the square root of time when cumulative volume versus square root time was plotted for experiments results. These experiments were dealing with a short samples, small diameters, and single linear flow systems.

When the square root of time was used to analyze the experiments results, neither a good fitting nor an analysis explanation for all of the curves because the shapes were smaller with a downward trend. This study attempted to use semi-log plots. Semi-log plots couldn't explain the core damage since the curves trend was upward with same shapes. This study also attempted to use poloymeteric equations. Analysis of these equations was not explaining the experiments results. This study used a linear analytical model relationship between cumulative volumes versus filtration time with a good fits result. This work will analyze the experiments results of low permeability sandstone core samples to evaluate the effectiveness of various preformed particle gels (PPGs) with different brine concentrations and particle sizes.

\section{RESEARCH METHODOLOGY}

\section{Experimental Materials}

Different materials have been used to accomplish this research, including preformed particle gels (PPGs), various particle sizes, and sandstone cores.

\section{Preformed Particle Gels (PPGs)}

Two types of PPGs were selected for the experiments: Daqing (DQ) and LiquiBlockTM 40K (40K) gels. DQ is a strong gel particle with a higher elastic module after becoming fully swollen. LiquiBlockTM $40 \mathrm{~K}$ is a weak gel particle with a lower 


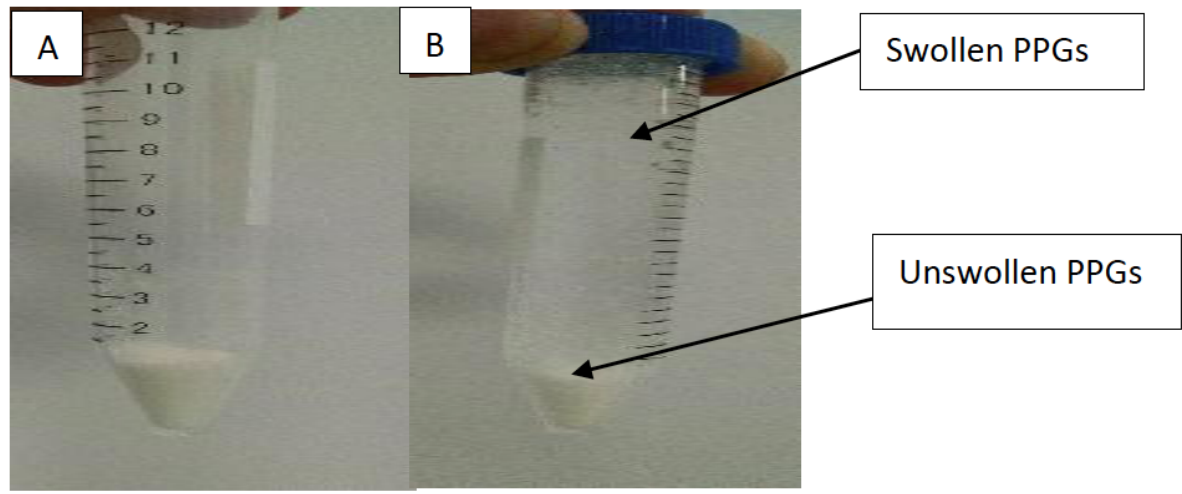

Figure 1: A) Unswollen PPGs in Graduated Centrifuge Tube. B) Partially Swollen PPGs in Graduated Centrifuge Tube.

elastic module after becoming fully swollen. The particle sizes for both gels were between 30 and 120 meshes.

\section{Swollen PPGs}

Figure 1 shows both PPGs before and after becoming swollen. The PPGs absorbed a large amount of water, increasing their volume.

\section{Swollen PPGs Sample Preparation}

The swollen PPGs used in these experiments was prepared as follows:

- An empty beaker was filled with a brine solution of the desired concentration to prepare the PPG.

- Depending on the concentration of the brine, (which was used to prepare the PPGs) grams of PPGs were weighed and slowly added to the brine solution.

- $\quad$ The sample was allowed to swell completely, a process that required more than 3 hours.

- The excess brine solution was separated from the swollen PPGs using a screen.

- The PPG was collected from the screen and stored.

- $\quad$ PPG full swollen weight was measured after extra water was removed.

\section{Brine}

Sodium chloride $(\mathrm{NaCl})$ was used to prepare all brines. Various brine concentrations at room temperature were selected to prepare the swollen PPGs. Brine concentration significantly affects the PPG swelling ratio and swollen particle strength. High salinity brine results in a lower swelling ratio and higher swollen particle strength. The brine viscosity was about $1 \mathrm{cp}$.

\section{Sandstone Core Samples}

Figure 2 shows a various sandstone cores which were collected to use in this research, with the permeability ranging from 5 to $320 \mathrm{mD}$. These sandstone cores were collected from three different sources: Missouri, St James, and Berea sandstone. The characteristics of each core, such as porosity $(\phi)$, permeability $(k)$, and average pore throat diameter $\left(d_{o}\right)$ were obtained [8-11]. The permeability ranges from 5 to $25 \mathrm{mD}$ were considered as a low permeable formation [8-11].

\section{Sandstone Core Samples Preparation}

The sandstone used in these experiments was prepared as follows:

- $\quad$ Several long sandstone cores were cut for the core flooding measurement.

- The dimensions of all short cores were $1.5 \mathrm{in}$ $(3.7 \mathrm{~cm})$ in length $(L)$ and 1.5 in. $(3.7 \mathrm{~cm})$ in diameter $(d)$, as shown in Figure 2. A caliper was used to ensure the dimension of each core the same for all experiments.

- The sandstone cores were put in an oven at 120 ${ }^{\circ} \mathrm{C}$ for $24 \mathrm{~h}$ before they were vacuumed and saturated to $100 \%$ with the desired brine. The core was vacuumed from the gas or air using the Soxhlet extractor, as shown in Figures $\mathbf{3}$ and $\mathbf{4}$.

\section{Procedure to Measure Core Porosity}

The procedures for the porosity measurements were as follows:

- $\quad$ Each core was cut from a different source and then the core dry weight $\left(W_{d}\right)$ was measured. 


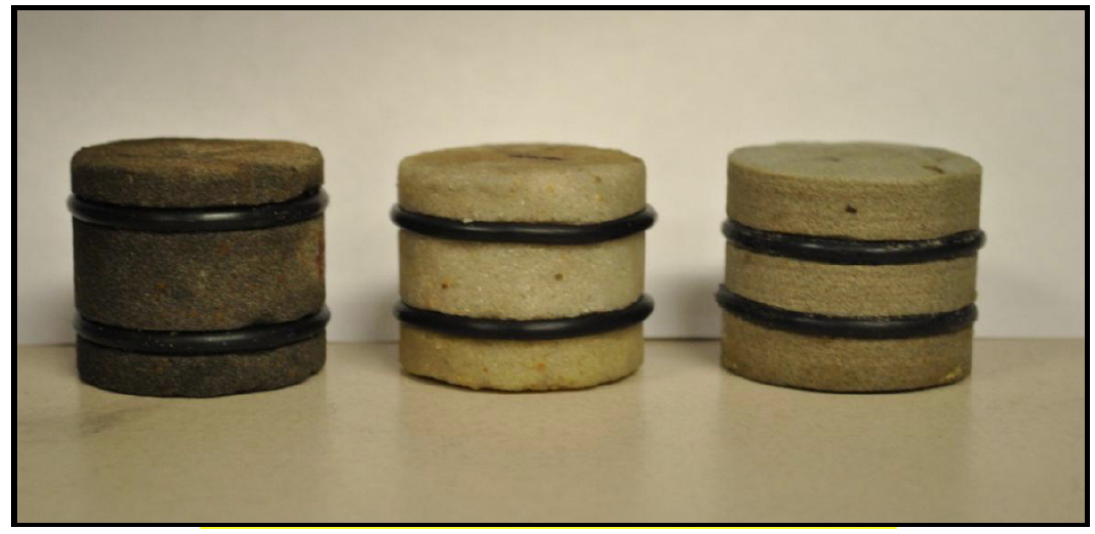

Figure 2: Sandstone Cores Prepared for Experiments.

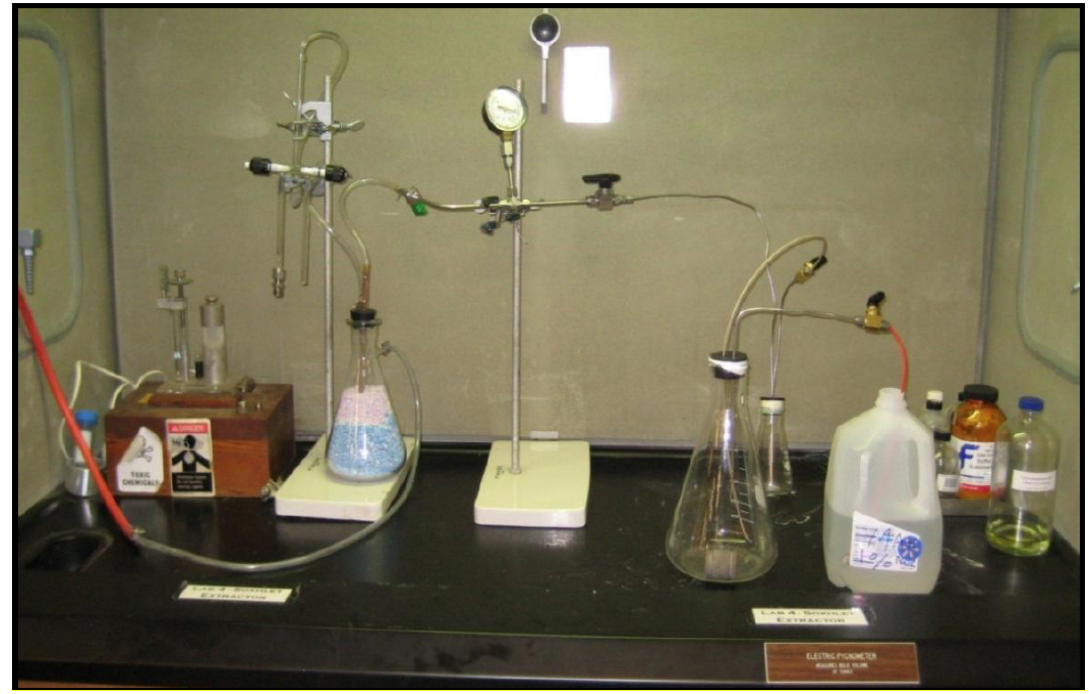

Figure 3: Vacuum Systems for Saturation of Model Brine with the Core Samples.

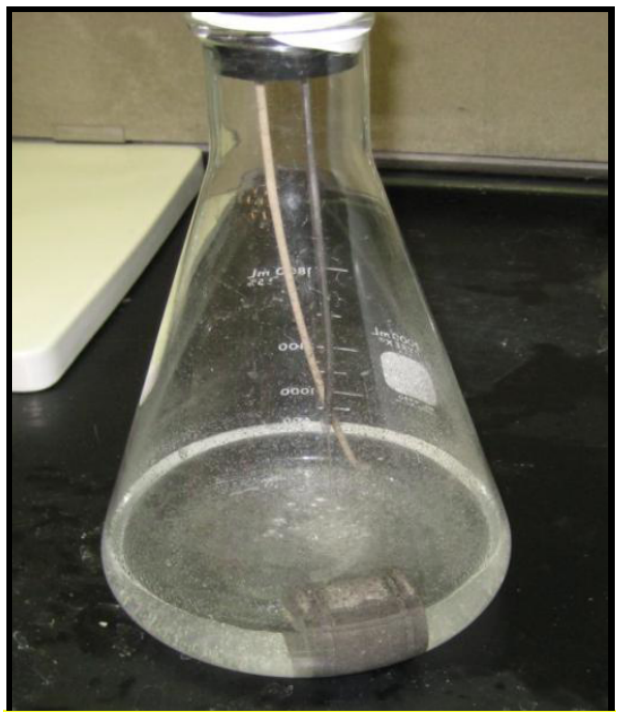

Figure 4: Core Vacuumed and Saturated with Brine.

- $\quad$ Both the core diameter $(d)$ and the core length $(L)$ were measured. The bulk volume $\left(V_{B}\right)$ was then calculated by using the following equation:

$$
V_{B}=\frac{\pi}{4} d^{2} l
$$

- The cores were dried and placed inside a beaker. The cap was closed and the buffer valve was opened and the desired brine valve was closed, as shown in Figure 3.

- $\quad$ The vacuum pump was turned on and the pressure gauge was observed until it reached 25 $\mathrm{Hg}$. If the cores had low permeability, it took a long time to reach the desired pressure.

- $\quad$ The buffer valve was closed and the brine valve was opened then the pump was turned off. It was important to make sure that the brine flowing into the beaker and the samples was saturated.

- $\quad$ After the cores were dried, vacuumed, and saturated, they were then weighed to measure 
the core saturated weight $\left(W_{s}\right)$, at room temperature.

- The brine density $\left[(\rho) 1.004879 \mathrm{gram} / \mathrm{cm}^{3}\right]$ was used to calculate the pore volume $\left(V_{P}\right)$ by using the following equations:

Brine weight $\left(B_{w}\right)=W_{s}-W_{d}$

$V_{P}=\frac{\text { brine weight }}{\text { brine density }}$

- $\quad$ The core's porosity $(\phi)$ was calculated by using the following equation:

$\operatorname{Porosity}(\phi)=\frac{V_{P}}{V_{B}} * 100$

\section{Filtration Test Model}

Different constant injection pressures were used to obtain filtration curves. Both filtration test (the relationship between the cumulative filtration volume vs. time) and the permeability measurement of each core both before and after gel treatment were used to confirm whether or not the PPG damaged the cores. The damage of the core could be determined from the shape of the curve. The effect of particle size, rock permeability, and brine concentration on core damage was investigated. Results of these filtration test experiments yielded information useful for promoting a best PPG treatment for conformance control in mature reservoirs. These results can be used to optimize PPG's design and, thus, prevent damage to the unswept, low-permeable zones/areas.

\section{Equipment}

The equipment used to perform these experiments was as follows:

- A Teledyne ISCO model 500D syringe pump.

- Filtration models composed of both a core sample and a round tube.

- $\quad$ Two O-rings made of plastic.

- Two caps made of fiber glass, one connected to the pump, and another one to the bottom of the round tube.

- $\quad$ Nuts and washers to tighten the apparatus.

- $\quad$ Four metal rods are used to place the round tube with top and bottom caps and tightening them.

\section{Experimental Setup}

Figures 5 represents the experimental setup, which was mainly composed of one Teledyne ISCO model 500D syringe pump (used for brine injection) and one filtration model. The filtration model constitutes a transparent round tube with a core sample fitted inside using two O-rings. Bolts were used to tighten two cups: one above the round tube and one under the round tube. This tube was then connected to the filtration model. Nuts and shims were used to fasten caps to the round tube and control the model. A hole on one side of the round tube acted as an inlet for the injection brine. On the other side of this round tube, another hole acted as an outlet to discharge the brine. Pressure

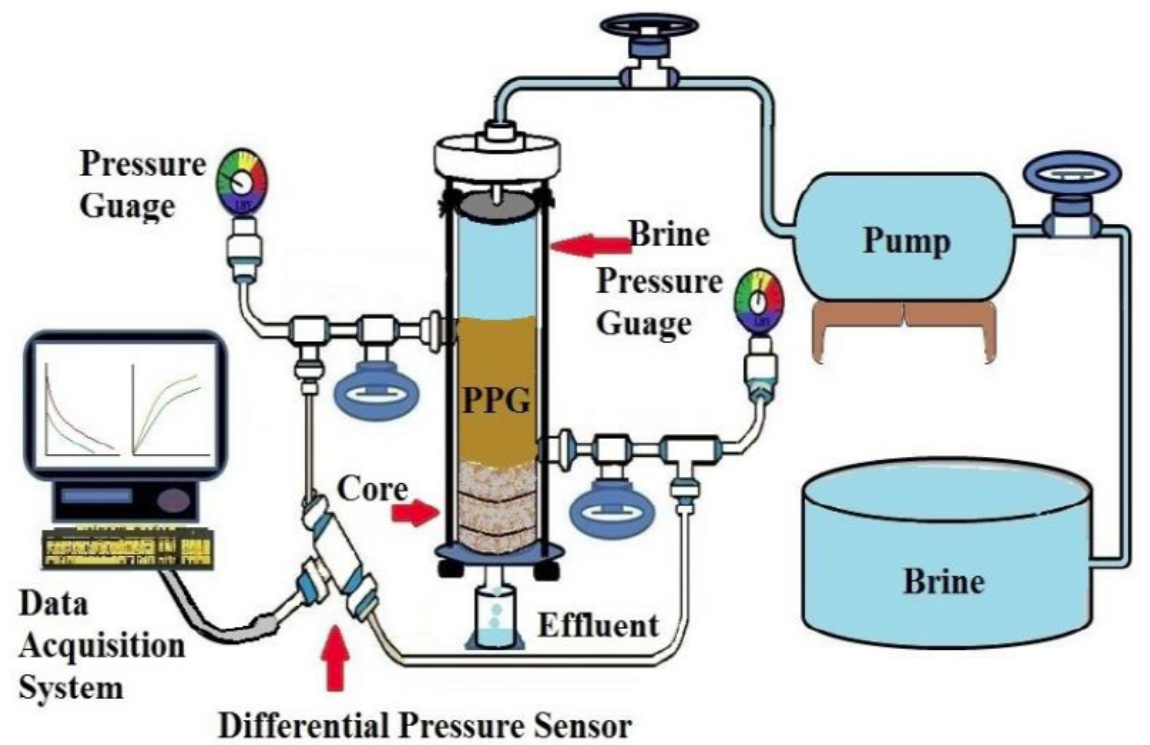

Figure 5: Filtration Test Model Schematic. 
gauges were connected at the top of both core samples and PPGs to record the pressures. A differential pressure sensor was connected to the data acquisition system to record the differential pressure along the PPG pack.

\section{Experimental Procedure}

The procedures for the filtration test experiments were briefly described as follows:

1. Core samples were vacuumed and saturated with brine, and porosity $(\phi)$ was obtained for each core.

2. The core sample was fitted on the bottom part of the transparent filtration model.

3. Brine was injected into the model to measure the rock permeability before gel treatment.

4. The completely swollen PPG was poured into the transparent tube, sitting on top of the core; the other space was filled with brine.

5. Brine was injected at pressures of $10,50,10$, $100,10,200,10,400$, and 10 psi, and each constant pressure was run either for $30 \mathrm{~min}$, or until $500 \mathrm{~mL}$ brine (pump capacity limitation) was pumped through the core. The purpose for repeatedly using the $10 \mathrm{psi}$ pressure was to determine whether the core was damaged further when the injection pressure increased. Accumulative effluent was recorded at 1, 2, 3, 4, $5,7.5,10,15,20,25$, and $30 \mathrm{~min}$ for the period of each pressure used.

6. PPG was poured out from the tube and brine was injected to measure rock permeability.

7. The above procedure was repeated for each experiment and the cumulative filtration volumes were plotted vs. time.

\section{RESULTS AND DISCUSSIONS}

\section{Effect of Particle Size}

Various particle sizes were prepared and used according to the procedures described in the filtration test section to establish the effect of particle size on core damage [8-11]. Figures 5 through 11 illustrate the cumulative filtration volume as a function of time for the filtration test experiments. Figures 5 through 11 present the effect of various particle sizes on core damage of cores with the original permeabilities of $5-320 \mathrm{mD}$.
Figures 5 through 11 summarize the results for each injection pressure curve using 30,50-60, 80, and 100-120 meshes PPG. The curves for both first 10 psi and 50 psi, shown in Figures 5 through 11, are not linear. The flow rate decreased with time, which indicates the swollen particles, damaged the porous media. The other curves in these Figures are straight lines, which means that flow rate does not change with time and, thus, no further damage occurred at higher pressures. The shapes of 10 psi curves also demonstrate that the core was damaged at first 10 psi and 50 psi pressure but no more damage occurred when the injection pressure increased further, because the 10 psi curves are overlaid except for the first 10 psi curve.

The results also determined the effect of particle size for 100-120 meshes on the core damage with a permeability of 5-320 $\mathrm{mD}$. The lines of injection pressure for each $10 \mathrm{psi}$ are not the same. The lines at 50,100 , and 200 psi are not in a straight line, except for the one at $400 \mathrm{psi}$, which indicates that the damage occurred when pressures of 50, 100, and 200 psi were used, and no more damage occurs when the pump pressure increased up to 400 psi.

\section{Effect of Brine Concentration}

Various brine concentrations were used to determine the effect of brine concentration on core damage with the permeability ranges of $(5-320 \mathrm{mD})$ [811]. Swollen PPG was prepared with a particle size of 30 mesh and brine concentrations of $0.05,1$, and $10 \%$ wt $(\mathrm{NaCl})$. As also shown in Figures 5 through 11, lower brine concentrations caused more damage than higher brine concentrations. This is because swollen PPGs prepared with a lower brine concentration are softer and penetrate into the porous media more easily than stronger PPGs prepared with a higher brine concentration.

Figures 5 through 11 also illustrate the cumulative filtration volume as a function of time for the filtration test experiments with various brine concentrations. These experiments were conducted to study the effect of brine concentration on the core damage In summary, the damage of different cores first occurred at first 10 psi and at 50 psi, even when using low brine concentrations. All filtration curves are a straight line for rock permeabilities with one exception. The lines for both first 10 psi and 50 psi are nonlinear. No further damage occurred, even as the injection pressure increased. 


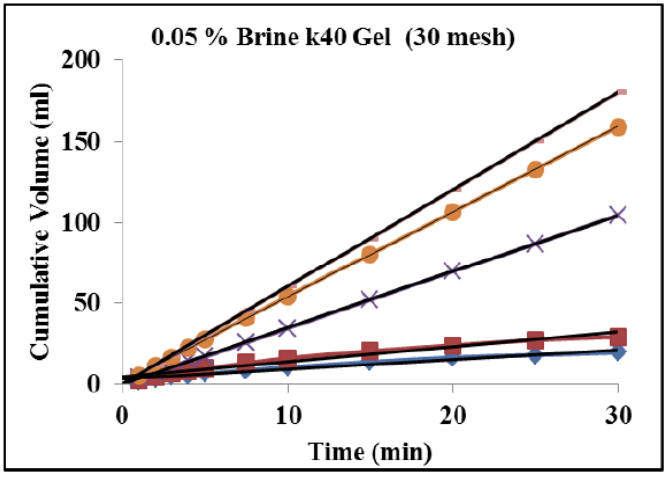

(a)

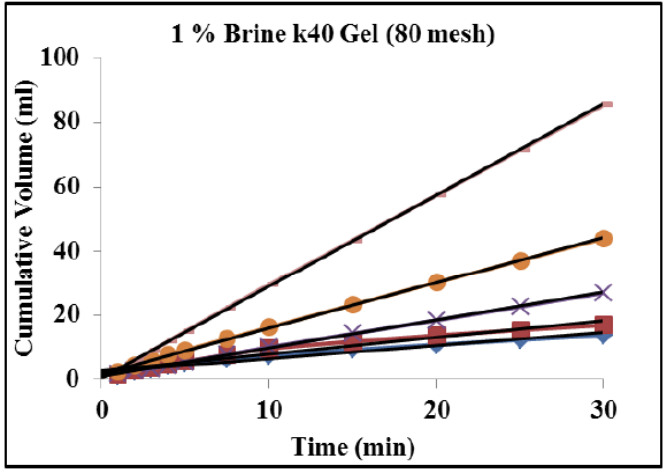

(c)

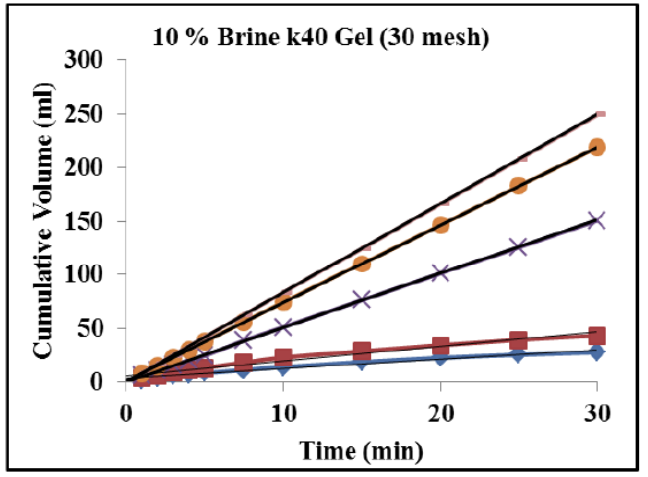

(b)

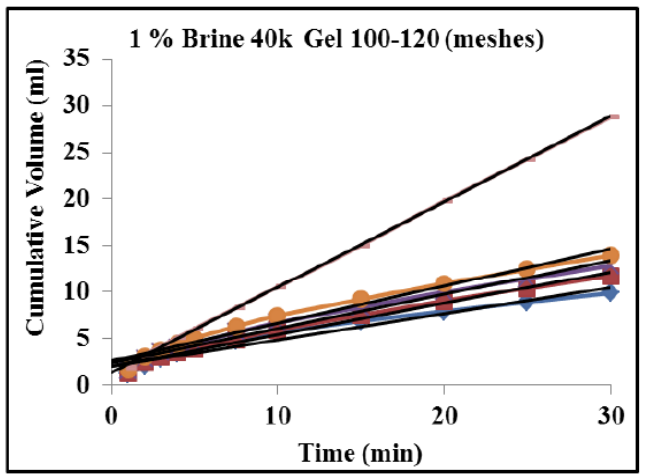

(d)

Figure 6: Typical Filtration Curves for Liquiblock ${ }^{\mathrm{TM}} 40 \mathrm{~K}$ Gel with $5-25 \mathrm{mD}$ : a) $1 \%$ Brine with 30 mesh b) $10 \%$ Brine with 30 mesh c) $1 \%$ Brine with 80 mesh d) $1 \%$ Brine with 100-120 meshes.

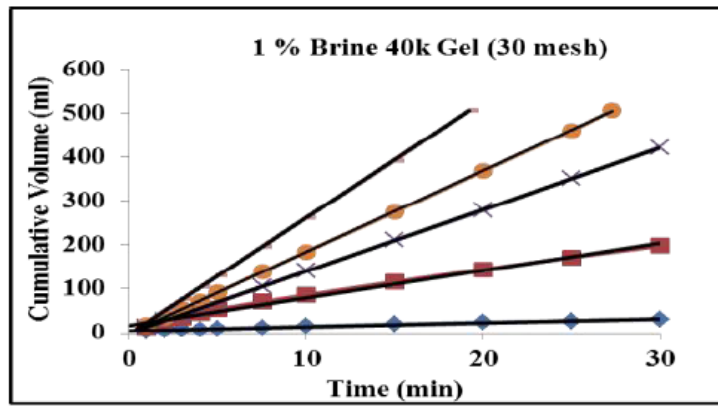

(a)

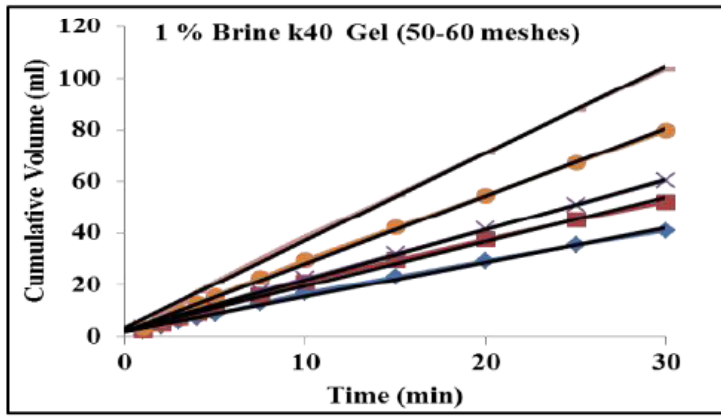

(c)

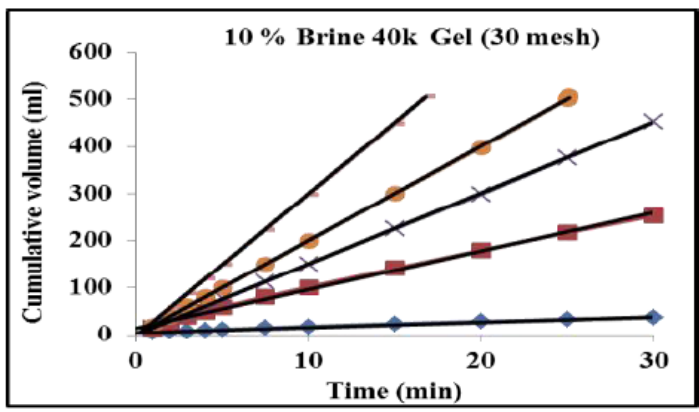

(b)

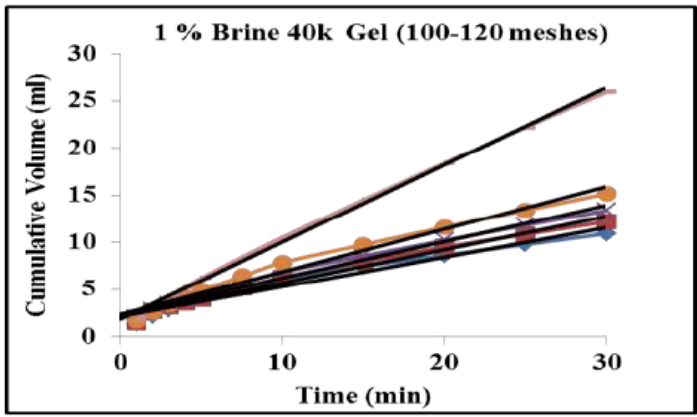

(d)

Figure 7: Typical Liner Curves Analytical Model for Liquiblock ${ }^{\mathrm{TM}}$ 40K Cel with 100-120 mD: (a) 1\% Brine with 30 mesh (b) $10 \%$ Brine with 30 mesh (c) $1 \%$ Brine with 50-60 meshes (d) $1 \%$ Brine with 100-120 meshes. 


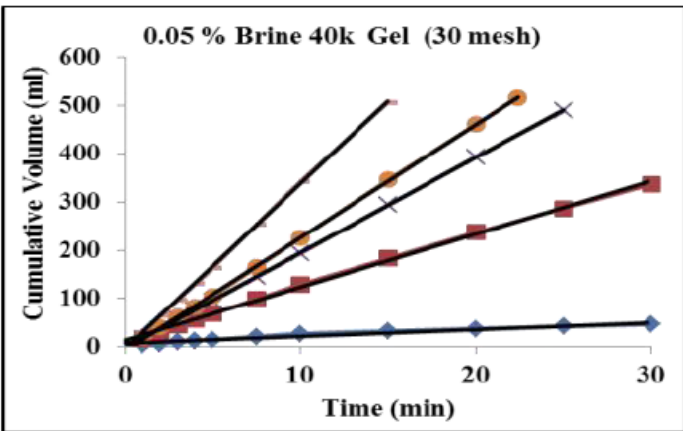

(a)

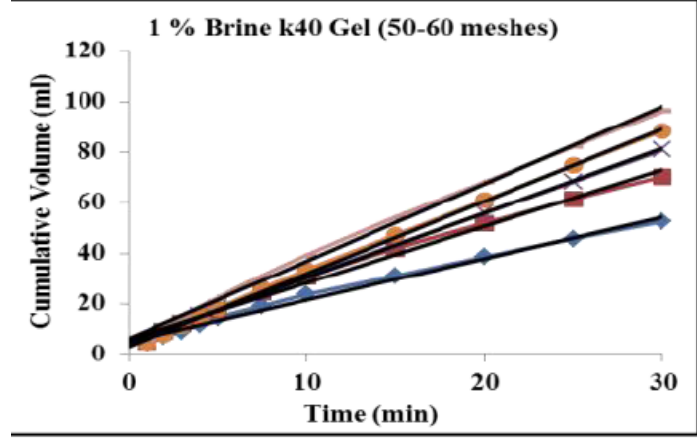

(c)

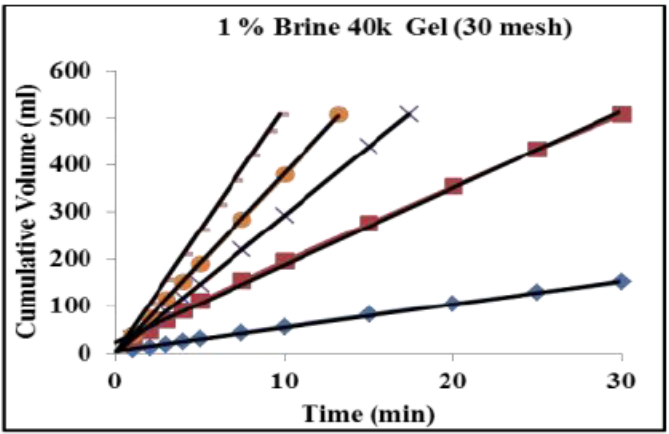

(b)

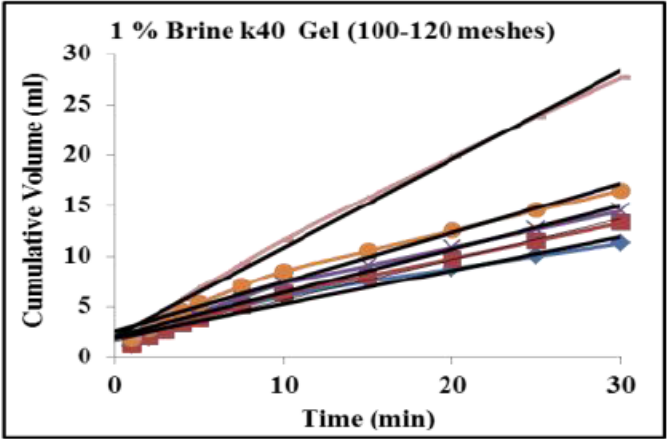

(d)

Figure 8: Typical Liner Curves Analytical Model for Liquiblock ${ }^{\mathrm{TM}} 40 \mathrm{~K}$ Gel with 290-320 mD: (a) $0.05 \%$ Brine with 30 mesh (b) 1 $\%$ Brine with 30 mesh (c) 1\% Brine with 50-60 meshes (d) 1\% Brine with 100-120 meshes.

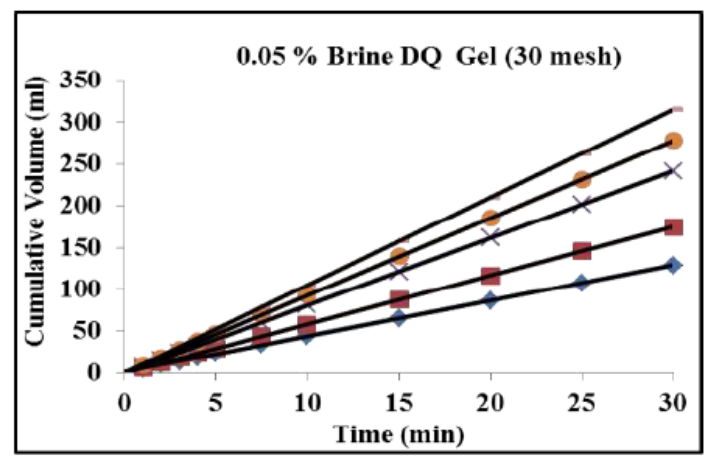

(a)

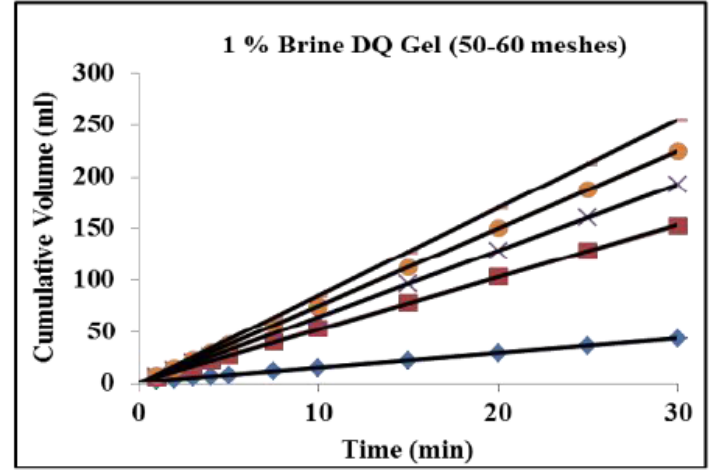

(c)

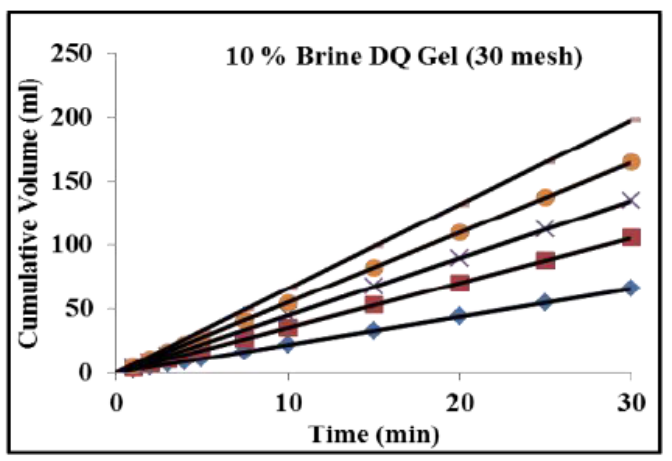

(b)

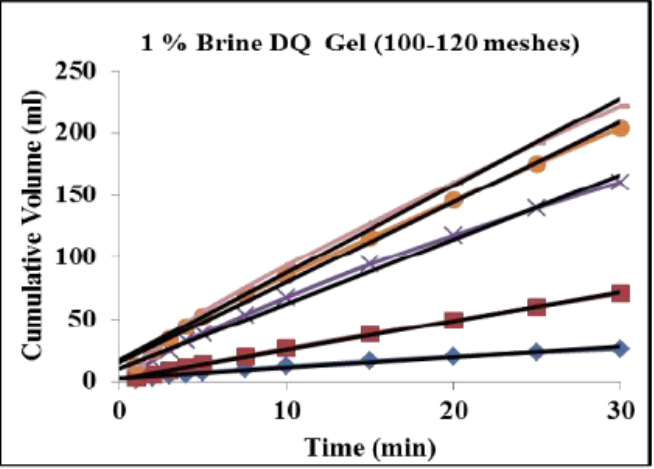

(d)

Figure 9: Typical Liner Curves Analytical Model for DQ Gel with 5-25 mD: (a) 0.05\% Brine with 30 mesh (b) $10 \%$ Brine with 30 mesh (c) $1 \%$ Brine with 50-60 meshes (d) $1 \%$ Brine with 100-120 meshes. 


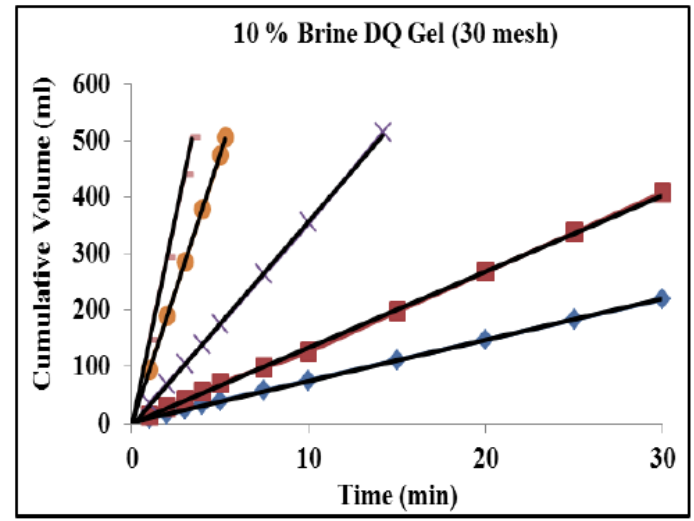

(a)

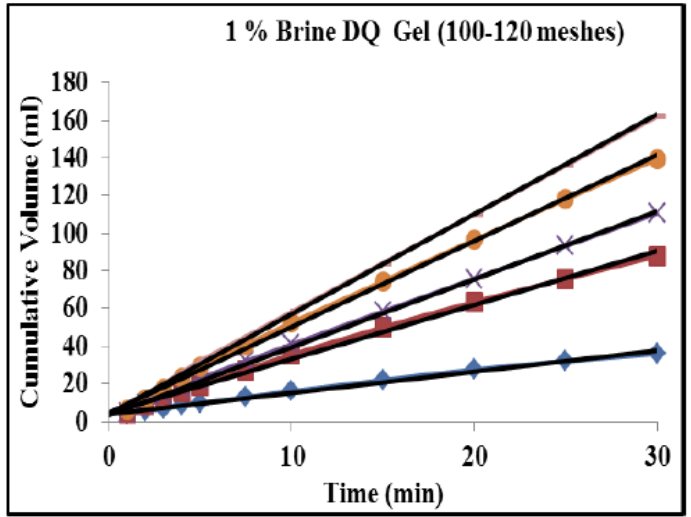

(b)

Figure 10: Typical Liner Curves Analytical Model for DQ Gel with 100-120 mD: (a) 10\% Brine with 30 mesh (b) $1 \%$ Brine with 100-120 meshes.

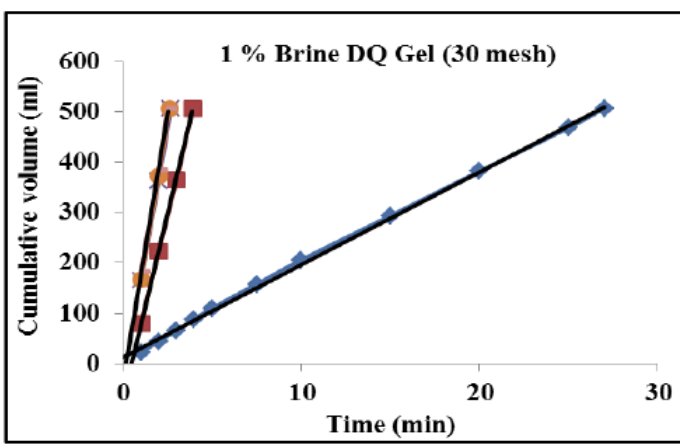

(a)

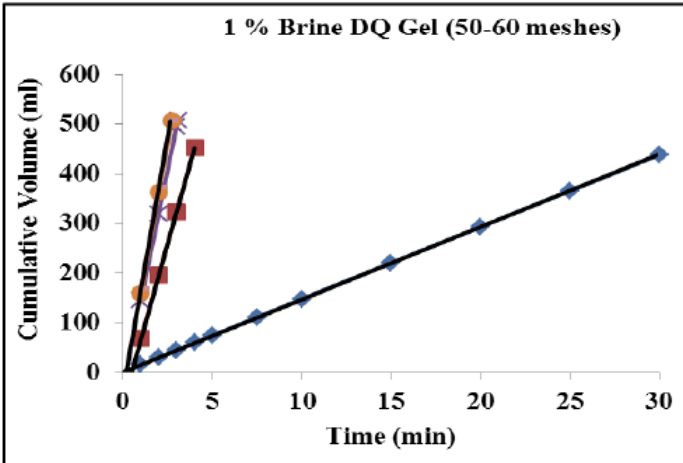

(c)

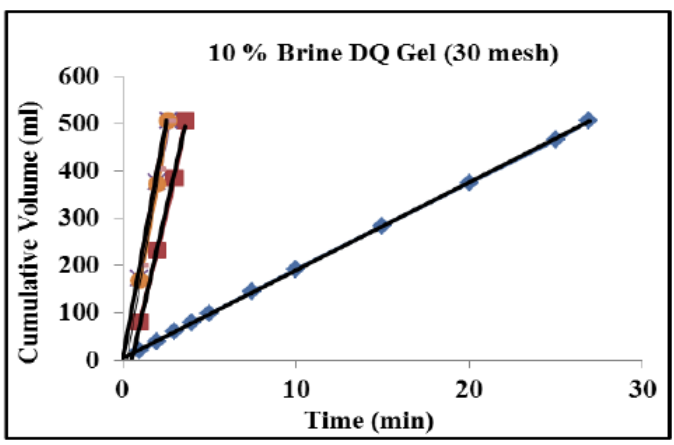

(b)

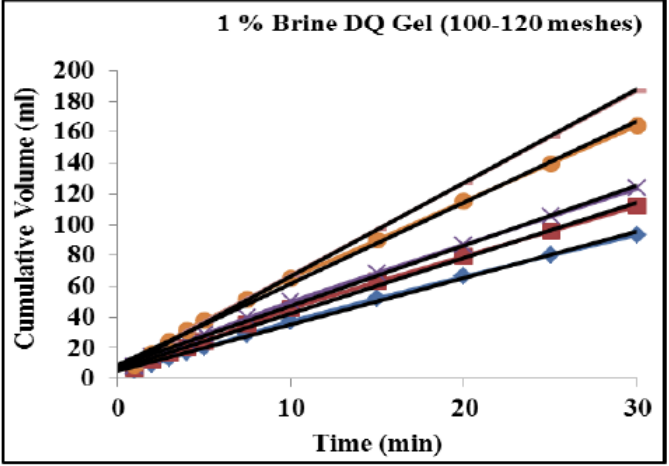

(d)

Figure 11: Typical Liner Curves Analytical Model for DQ Gel with 290-320 mD: (a) 1\% Brine with 30 mesh (b) $10 \%$ Brine with 30 mesh (c) $1 \%$ Brine with 50-60 meshes (d) 1\% Brine with 290-320 meshes.

The flow rate for the PPG prepared with a low brine concentration was lower than the sample prepared with a high brine concentration. PPG prepared with a low brine concentration is a weak gel and slightly compressed more which decrease the gel pack permeability.

The swollen particle sizes are larger at a low brine concentration than at a high brine concentration.
Experimental results suggest that the weak gels with a low brine concentration are softer and more deformable than those with a high brine concentration. Therefore, low brine concentration caused more core damage.

\section{Effect of the Rock Permeability}

Three cores with the permeability ranges of $5-25$, $100-120$, and $290-320 \mathrm{mD}$, were used to examine the 
effect of rock permeability on core damage. Permeability was measured after PPG was removed from the round tube with the flow rates of 1,2 , and 3 $\mathrm{mL} / \mathrm{min}$. Core permeabilities were reduced from $24.00 \%$ to $36.89 \%$ by using particle sizes of $30,50-60$, and 80 meshes for low-permeability rocks. In contrast, a particle size of 100-120 meshes reduced their permeabilities from $64.76 \%$ to $99.30 \%$ and, thus, damaged the core more. The permeability reduction also increased with the increase of rock permeability. The dependence of core damage on particle size and rock permeability is caused by the relationship of particle size, rock permeability, and particle penetration into rocks. When high core permeability and small particle were used, more PPGs penetrate into the rocks.

\section{Effect of Gel Types}

Liquiblock $^{\mathrm{TM}} 40 \mathrm{~K}$ gel is a weak gel compare with $\mathrm{DQ}$ gel. DQ gel is a stronger gel with higher elastic model. $40 \mathrm{~K}$ gel with larger particle sizes adsorb more water than a smaller particle sizes. During filtration tests, the weak gels with larger particle sizes and low brine concentrations compressed further in the fluid channels and loss more water than stronger gel with small particle sizes and higher brine concentrations. As a results more gel deformations occurred and caused a shift in the $y$-axis which can be seen in Figures 6 through 11, especially when the 100-120 meshes and $0.05 \%$ brine concentrations were applied.

\section{Quantitative Analytical}

Linear curve equations for the best fitting equation as you can see in the Figures 6 through 11, the fitting equation for the all curves show in the Table 1 through 6.

When core damage occurred, the filtration volume curves for static filtration tests required a few minutes to become a linear function of the time. According to quantitative analytical model for all of our filtration tests, the cumulative filtration test volume is explained by the following equation:

$\mathrm{V}_{\mathrm{cf}}=\mathrm{mt}+\mathrm{b}$

Table 1: Shows the Quantitative Analytical Model Equations for Liquiblock ${ }^{\mathrm{TM}}$ 40K Gel with Core Permeability of 5-25 $\mathrm{mD}$ for Various Particle Sizes and Brine Concentrations

\begin{tabular}{|c|c|c|c|c|}
\hline Particle Size (mesh) & $\mathrm{NaCl} \%$ & Pressure (psi) & Fitting Equation & $\mathbf{R}^{2}$ \\
\hline \multirow[t]{5}{*}{30} & \multirow[t]{5}{*}{0.05} & 10 & $V=0.6053 t+3.3299$ & 0.9638 \\
\hline & & 50 & $V=0.9129 t+4.672$ & 0.9627 \\
\hline & & 100 & $V=3.4697 t+0.2552$ & 0.9999 \\
\hline & & 200 & $V=5.2797 t+1.062$ & 1 \\
\hline & & 400 & $V=6 t+0.25$ & 1 \\
\hline \multirow[t]{5}{*}{30} & \multirow[t]{5}{*}{10} & 10 & $V=0.8675 t+4.2124$ & 0.9741 \\
\hline & & 50 & $V=1.3495 t+6.2147$ & 0.9709 \\
\hline & & 100 & $V=5.0218 t+0.7348$ & 0.9999 \\
\hline & & 200 & $V=7.2757 t+1.2437$ & 1 \\
\hline & & 400 & $V=8.302 t+0.3097$ & 1 \\
\hline \multirow[t]{5}{*}{80} & \multirow[t]{5}{*}{1} & 10 & $V=0.4054 t+2.4853$ & 0.9615 \\
\hline & & 50 & $V=0.5212 t+2.7319$ & 0.9559 \\
\hline & & 100 & $V=0.8689 t+1.037$ & 0.9996 \\
\hline & & 200 & $V=1.4184 t+1.7904$ & 0.9994 \\
\hline & & 400 & $V=2.8396 t+0.6267$ & 0.9999 \\
\hline \multirow[t]{5}{*}{$100-120$} & \multirow[t]{5}{*}{1} & 10 & $V=0.2804 t+2.1115$ & 0.9552 \\
\hline & & 50 & $V=0.3396 t+2.0403$ & 0.9842 \\
\hline & & 100 & $V=0.369 t+2.3905$ & 0.9804 \\
\hline & & 200 & $V=0.401 t+2.6657$ & 0.9752 \\
\hline & & 400 & $V=0.9184 t+1.341$ & 0.9997 \\
\hline
\end{tabular}


Table 2: Shows the Quantitative Analytical Model Results Liner Equations Liquiblock TM 40K gel with Core Permeability of 100-120 mD for Various Particle Sizes and Brine Concentrations

\begin{tabular}{|c|c|c|c|c|}
\hline Particle Size (mesh) & Brine Concentration (\%) & Pressure (psi) & Fitting Equation & $\mathbf{R}^{2}$ \\
\hline \multirow[t]{5}{*}{30} & \multirow[t]{5}{*}{1} & 10 & $V=0.9518 t+3.9823$ & 0.9849 \\
\hline & & 50 & $V=6.2262 t+17.708$ & 0.9914 \\
\hline & & 100 & $V=14.039 t+0.1579$ & 1 \\
\hline & & 200 & $V=18.552 t-1.8127$ & 1 \\
\hline & & 400 & $V=26.379 t-1.1825$ & 1 \\
\hline \multirow{4}{*}{30} & \multirow{4}{*}{10} & 50 & $V=8.1346 t+14.637$ & 0.9967 \\
\hline & & 100 & $V=15.039 t+0.1579$ & 1 \\
\hline & & 200 & $V=20.082 t-0.8346$ & 0.9999 \\
\hline & & 400 & $V=30.138 t-1.703$ & 1 \\
\hline \multirow[t]{2}{*}{$50-60$} & \multirow[t]{2}{*}{1} & 10 & $V=1.3416 t+2.0352$ & 0.9963 \\
\hline & & 400 & $V=3.3885 t+3.4377$ & 0.9986 \\
\hline \multirow[t]{5}{*}{$100-120$} & \multirow[t]{5}{*}{1} & 10 & $V=0.3139 t+2.1768$ & 0.9656 \\
\hline & & 50 & $V=0.3546 t+2.1567$ & 0.9826 \\
\hline & & 100 & $V=0.3842 t+2.3801$ & 0.9877 \\
\hline & & 200 & $V=0.4529 t+2.3416$ & 0.9791 \\
\hline & & 400 & $V=0.8185 t+1.8896$ & 0.9975 \\
\hline
\end{tabular}

Table 3: Shows the Quantitative Analytical Model Results Liner Equations Liquiblock ${ }^{\mathrm{TM}}$ 40K Gel with Core Permeability of 290-320 mD for Various Particle Sizes and Brine Concentrations

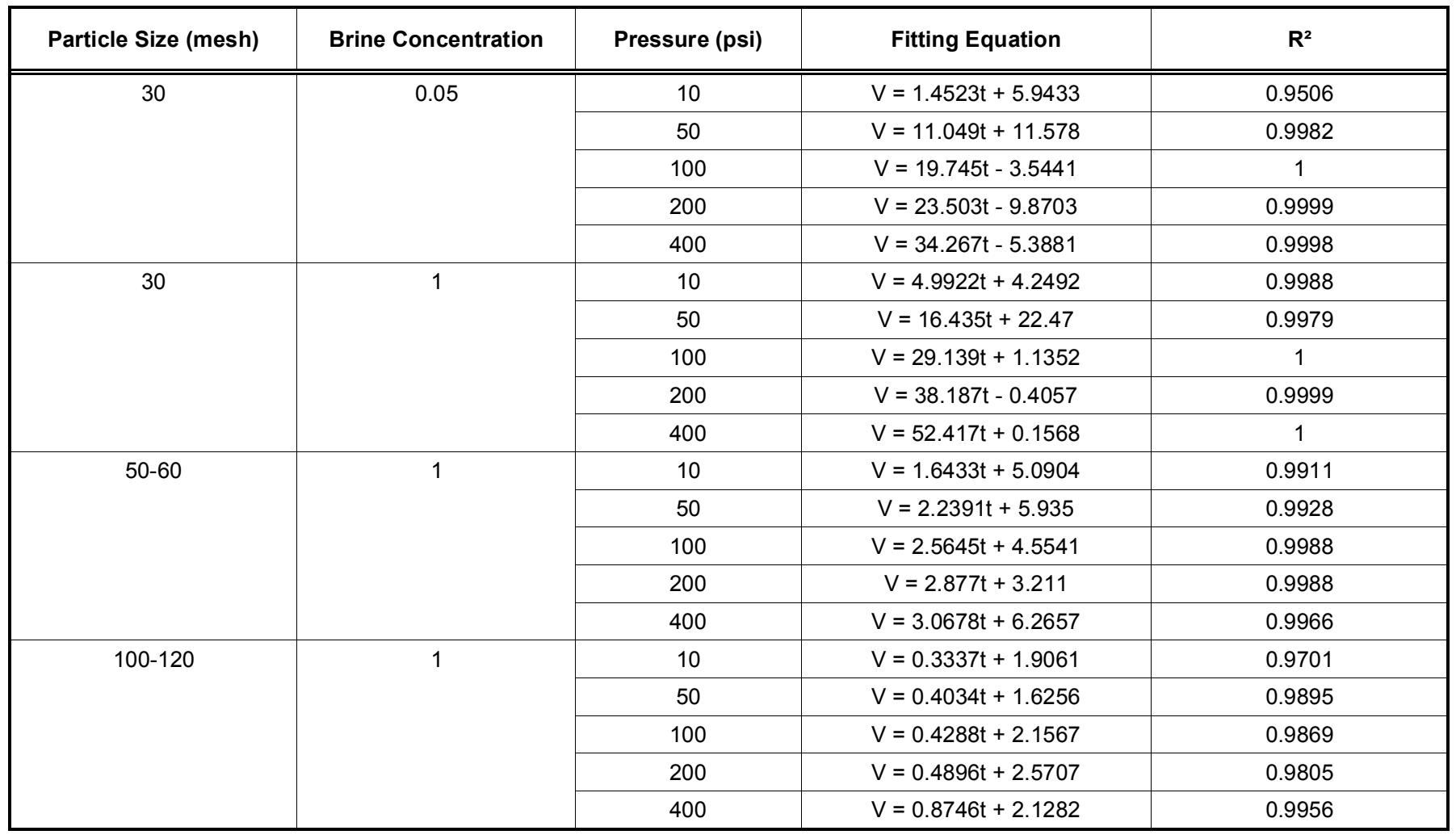


Table 4: Shows the Quantitative Analytical Model Results Liner Equations DQ Gel with Core Permeability of 5-25 mD for Various Particle Sizes and Brine Concentrations

\begin{tabular}{|c|c|c|c|c|}
\hline Particle Size (mesh) & Brine Concentration & Pressure (psi) & Fitting Equation & $\mathbf{R}^{2}$ \\
\hline \multirow[t]{5}{*}{30} & \multirow[t]{5}{*}{0.05} & 10 & $V=4.2701 t+0.9464$ & 0.9999 \\
\hline & & 50 & $V=5.8084 t+0.0429$ & 1 \\
\hline & & 100 & $V=8.0679 t-0.1105$ & 1 \\
\hline & & 200 & $V=9.2589 t+0.14$ & 1 \\
\hline & & 400 & $V=10.5 t$ & 1 \\
\hline \multirow[t]{5}{*}{30} & \multirow[t]{5}{*}{1} & 10 & $V=3.1817 t-0.6141$ & 1 \\
\hline & & 50 & $V=4.6267 t+0.557$ & 1 \\
\hline & & 100 & $V=5.8436 t+0.4783$ & 1 \\
\hline & & 200 & $V=6.9 t$ & 1 \\
\hline & & 400 & $V=7.8 \mathrm{t}$ & 1 \\
\hline \multirow[t]{5}{*}{30} & \multirow[t]{5}{*}{10} & 10 & $V=2.2 \mathrm{t}$ & 1 \\
\hline & & 50 & $V=3.5213 t+0.0127$ & 1 \\
\hline & & 100 & $V=4.5 t-0.1$ & 1 \\
\hline & & 200 & $V=5.5 t$ & 1 \\
\hline & & 400 & $V=6.6 t$ & 1 \\
\hline \multirow[t]{5}{*}{$50-60$} & \multirow[t]{5}{*}{1} & 10 & $V=1.4043 t+1.2609$ & 1 \\
\hline & & 50 & $V=5.0672 t+1.5313$ & 0.9999 \\
\hline & & 100 & $V=6.4105 t+0.4281$ & 1 \\
\hline & & 200 & $V=7.501 t+0.1796$ & 1 \\
\hline & & 400 & $V=8.501 t+0.2796$ & 1 \\
\hline \multirow[t]{5}{*}{80} & \multirow[t]{5}{*}{1} & 10 & $V=1.222 t+1.473$ & 0.9997 \\
\hline & & 50 & $V=4.0672 t+1.7313$ & 0.9998 \\
\hline & & 100 & $V=5.1324 t+1.7069$ & 0.9997 \\
\hline & & 200 & $V=6.501 t+0.1796$ & 1 \\
\hline & & 400 & $V=7.5 \mathrm{t}$ & 1 \\
\hline \multirow[t]{5}{*}{$100-120$} & \multirow[t]{5}{*}{1} & 10 & $V=0.8293 t+2.8354$ & 0.9842 \\
\hline & & 50 & $V=2.3431 t+2.0654$ & 0.9987 \\
\hline & & 100 & $V=5.2105 t+10.772$ & 0.9928 \\
\hline & & 200 & $V=6.4458 t+15.864$ & 0.9944 \\
\hline & & 400 & $V=7.0541 t+17.127$ & 0.9941 \\
\hline
\end{tabular}

Where, $V_{\text {cf }}$ is the cumulative filtration volume, $m$ is the slop of the linear curve, $t$ is the filtration time, and $b$ is the intercept of the linear curve.

Quantitative analytical model results showed the value of the slop $m$ increases as the injection pressure increases. Compared with the experiments results, Tables 1 through 6 illustrate that, if the value of the intercept $b>2$ the damage occurred because the gel particles invasion started into the core surface. Results from the quantitative analytical model were indicated to have a good fitting with almost all of the experimental results. The results of this work were conducted by using a very clean sandstone samples. The core samples did not contain clays. Zhou et al. (1997) [20] reported the formation damage which was caused by clay swelling.

According to our analytical results, the PPGs lost some water (volume lost). The value of water loss could explain the core damage. These water losses typically occurred at first injection pressures. The shift 
Table 5: Shows the Quantitative Analytical Model Results Liner Equations DQ Gel with Core Permeability of 100-120 $\mathrm{mD}$ for Various Particle Sizes and Brine Concentrations

\begin{tabular}{|c|c|c|c|c|}
\hline Particle Size (mesh) & Brine Concentration & Pressure (psi) & Fitting Equation & $\mathbf{R}^{2}$ \\
\hline \multirow[t]{3}{*}{30} & \multirow[t]{3}{*}{10} & 10 & $V=7.2733 t+2.4563$ & 0.9998 \\
\hline & & 100 & $V=36.251 \mathrm{t}-4.1154$ & 0.9997 \\
\hline & & 200 & $V=95.353 t-0.7789$ & 0.9999 \\
\hline \multirow[t]{4}{*}{$100-120$} & \multirow[t]{4}{*}{1} & 10 & $V=1.1287 t+3.7965$ & 0.9929 \\
\hline & & 50 & $V=2.8852 t+4.165$ & 0.9955 \\
\hline & & 100 & $V=3.6056 t+3.4918$ & 0.9988 \\
\hline & & 200 & $V=4.5652 t+4.5052$ & 0.9984 \\
\hline
\end{tabular}

Table 6: Shows the Quantitative Analytical Model Results Liner Equations DQ Gel with Core Permeability 290-320 mD for Various Particle Sizes and Brine Concentrations

\begin{tabular}{|c|c|c|c|c|}
\hline Particle Size (mesh) & Brine Concentration & Pressure (psi) & Fitting Equation & $\mathbf{R}^{2}$ \\
\hline \multirow[t]{3}{*}{30} & \multirow[t]{3}{*}{0.05} & 10 & $V=20.155 t+8.5524$ & 0.9993 \\
\hline & & 100 & $V=199.78 t-20.591$ & 0.9997 \\
\hline & & 200 & $V=204.43 t-16.544$ & 1 \\
\hline \multirow[t]{4}{*}{30} & \multirow[t]{4}{*}{1} & 10 & $V=18.403 t+13.165$ & 0.9989 \\
\hline & & 50 & $V=146.3 t-69.089$ & 0.9994 \\
\hline & & 100 & $V=203.08 t-39.833$ & 0.9995 \\
\hline & & 200 & $V=211.84 t-46.429$ & 0.9995 \\
\hline \multirow{3}{*}{30} & \multirow{3}{*}{10} & 100 & $V=196.44 t-20.233$ & 0.9992 \\
\hline & & 200 & $V=211.07 t-45$ & 0.9993 \\
\hline & & 400 & $V=199.37 t+1.8362$ & 0.9996 \\
\hline \multirow[t]{5}{*}{$50-60$} & \multirow[t]{5}{*}{1} & 10 & $V=14.567 t+1.4621$ & 1 \\
\hline & & 50 & $V=128 t-60$ & 1 \\
\hline & & 100 & $V=173.44 t-27.825$ & 0.9998 \\
\hline & & 200 & $V=198.59 t-36.973$ & 0.9996 \\
\hline & & 400 & $V=203.67 t-33.073$ & 1 \\
\hline
\end{tabular}


of the volume vertically in the $y$-intercept was occurred even no core damage because the PPGs lose some water. The fitting equations can be helped in the real applications to get an idea about the effect of the particle gels on the low permeable formation before starting gel treatments. The results can be used as a reference in the field applications. Tables 1 through 6 . Shows the quantitative analytical model results for both gels (Liquiblock ${ }^{\mathrm{TM}} 40 \mathrm{~K}$ gel and $\mathrm{DQ}$ gel) with different brine concentrations and particle sizes which indicate the intercept of each filtration test curve.

\section{CONCLUSIONS}

- It is the first time to use quantitative analytical model to analysis the formation damage by the PPGs.

- The relationship curves between the cumulative volume versus filtration time show that if the value of the curve intercept $b>2$ the gel will damage the formation.

- Water loss value from the particle gel can give an indication about the formation damage. Weak gels loss more water than strong gel. Therefor the particle sizes will be smaller and penetrate further through the reservoir rocks.

Preformed particle gels loss water at first injection pressures. The shift of the volume vertically in the $y$-intercept because the Preformed particle gels lose some water.

- $\quad$ PPG damage on rocks was affected by particle sizes and brine concentrations; more damage occurred with a small particle size (100-120 meshes) and a low brine concentration (0.05 wt $\% \mathrm{NaCl}$ ).

PPG damage on rocks was affected by core permeability; more damage occurred when a high-permeability rock of (290-320 mD) was used.

- $\quad$ The damage caused by PPGs on unswept, lowpermeability, oil-rich zones could be effectively controlled by controlling particle gel strength, gel type, particle size, and brine concentration.

- $\quad$ This research results can be used to properly select the gel particles that will not damage the formation for the best a particle gel treatment.

\section{ACKNOWLEDGEMENT}

The authors gratefully acknowledge the financial support given for this work by Research Partnership to Secure Energy for America (RPSEA). Also the authors would like to thank McCoy School of Engineering, Midwestern State University, Wichita Falls, Texas, USA, Petroleum Engineering Department at Missouri University of Science and Technology, Rolla, Missouri, USA, and the department of Petroleum Engineering, Sirte University, Sirte, Libya for their support.

\section{REFERENCES}

[1] Vetter OJ, Kandarpa V, Stratton M, Veith E. Particle invasion into porous medium and related injectivity problems. Proceedings of the SPE International Symposium on Oilfield Chemistry, San Antonio, Texas, USA, 4-6 February. SPE $1987 ; 16255$. https://doi.org/10.2118/16255-MS

[2] Ershaghi I, Hashemi R, Caothien SC, Abdassah D. Injectivity losses under particle cake buildup and particle invasion. Proceedings of the SPE California Regional Meeting, 2-4 April, Oakland, California, USA. SPE 1986; 15073. https://doi.org/10.2118/15073-MS

[3] Eylander JGR. Suspended Solids Specifications for Water Injection From Coreflood Tests. SPE Reservoir Eng 1988; 3(4): 1287-1294

https://doi.org/10.2118/16256-PA

[4] Al-Abduwani FH, Shirzadi A, Currie PK. Formation Damage vs. Solid Particles Deposition Profile During LaboratorySimulated Produced-Water Reinjection. SPE Journal 2005b; 10(2): 138-151. SPE-82235-PA. https://doi.org/10.2118/82235-PA

[5] Hsi D, Dudzik S, Lane H, Buettner W, Neira D. Formation Injectivity Damage Due to Produced Water Reinjection. Proceedings of the SPE Formation Damage Control Symposium, Lafayette, Louisiana, USA, 7-10 February, SPE 1994; 27395. https://doi.org/10.2118/27395-MS

[6] Coleman R, McLelland G. Produced Water Re-Injection: How Clean is Clean? Proceedings of the SPE Formation Damage Control Symposium, Lafayette, Louisiana, USA, SPE 1994; 27394.

https://doi.org/10.2118/27394-MS

[7] Ali M, Currie P, Salman M. The Effect of Residual Oil on Deep-Bed Filtration of Particles in Injection Water. SPE Prod \& Oper 2009; 24(1): 117-123. SPE-107619-PA, Doi:10. 2118/107619-PA.

[8] Elsharafi MO, Bai B. Effect of Weak Preformed Particle Ge on Unswept Oil Zones/Areas during Conformance Contro Treatments. Industrial \& Engineering Chemistry Research 2012; 51(35): 11547-11554. https://doi.org/10.2118/16256-PA

[9] Elsharafi M, Bai B. Effect of Strong Preformed Particle Gel on Unswept Oil Zones/Areas during Conformance Contro Treatments. Proceedings of the 75th EAGE Conference \& Exhibition incorporating SPE EUROPEC, London, United Kingdom, 2013; SPE-164879-MS. https://doi.org/10.2118/164879-MS

[10] Elsharafi M, Bai B. Minimizing formation damage for preformed particle gels treatment in mature reservoirs. SPE174645-MS to the SPE Asia Pacific Enhanced Oil Recovery Conference. 11-13 August, 2015 in Kuala Lumpur, Malaysia 2015. 
[11] Elsharafi MO, Bai B. Influence of strong preformed particle gels on low permeable formations in mature reservoirs. Pet Sci 2016; 13: 77-90. https://doi.org/10.1007/s12182-015-0072-3

[12] Outmans H. Mechanics of Static and Dynamic Filtration In the Borehole. SPE Journal 1963; 3(3): 236-244. SPE 491. https://doi.org/10.2118/491-PA

[13] Barkman HJ, Davidson DH. Measuring water quality and predicting well impairment, SOC. Petrol Engr JPT 1972; 24(7): 865-873. https://doi.org/10.2118/3543-PA

[14] Gulbis J, Dowell D. Dynamic Fluid Loss of Fracturing Fluids. Proceedings of the SPE Annual Technical Conference and Exhibition, San Francisco, California, 5-8 October. SPE 1983; 12154 https://doi.org/10.2118/12154-MS

[15] Roodhart L, Koninklijke. Fracturing fluids: fluid loss measurement under dynamic conditions. PPE Journal 1985; 25(5): 629-636. https://doi.org/10.2118/11900-PA
[16] Penny GS, Conway MW, Lee W. Control and Modeling of Fluid Leakoff During Hydraulic Fracturing. Journal of Petroleum Technology 1985; 37(6): 1071-1081. SPE 12486. https://doi.org/10.2118/12486-PA

[17] Bourgoyne A, Millheim K, Chenevert M, Young F. Applied Drilling Engineering Handbook, Society of Petroleum Engineering, Richardson, Texas, USA 1986.

[18] Chin W. Formation Invasion. Gulf Publishing Company 1995.

[19] Longeron D, Argillier J-F, Audibert A. An Intergrated Experimental Approach For Evaluating Formation Damage Due to Drilling and Completion Fluids. Proceedings of the European Formation Damage Conference, Hague, Netherlands, 15-16 May, SPE 1995; 30089. https://doi.org/10.2118/30089-MS

[20] Zhou ZJ, Cameron S, Kadatz B, Gunter WD. Clay Swelling Diagrams: Their Applications in Formation Damage Control. SPE Journal 1997; 2(02): 1997. SPE-31123-PA. https://doi.org/10.2118/31123-PA 